\title{
A four-layered model for flow of non-Newtonian fluid in an artery with mild stenosis
}

\author{
R PONALAGUSAMY and RAMAKRISHNA MANCHI*® \\ Department of Mathematics, National Institute of Technology, Tiruchirappalli 620015, India \\ e-mail: rpalagu@nitt.edu; manchinitt2017@gmail.com
}

MS received 22 July 2018; revised 10 April 2019; accepted 12 April 2019; published online 13 June 2019

\begin{abstract}
The present article deals with a four-layered mathematical model for blood flow through an artery with mild stenosis. The four-layered model comprises a cell-rich core of suspension of all the erythrocytes described as a non-Newtonian (Jeffrey) fluid, a peripheral zone of cell-free plasma (Newtonian fluid) and the stenosed artery with porous wall consisting of a thin transition (Brinkman) layer followed by Darcy region. Analytical expressions have been obtained for velocity profiles in all the four regions, total volumetric flow rate, wall shear stress and flow impedance. MATLAB software is employed to compute numerical values of the pressure gradient. The influences of different parameters such as variable core fluid viscosity, hematocrit, thickness of the plasma layer, Brinkman and Darcy layer thickness, Darcy number, Jeffrey fluid parameter, and size and shape parameters of stenosis on the physiologically vital flow characteristics, specifically velocity profile, volume flow rate, wall shear stress and flow impedance, have been examined. It is observed that the wall shear stress and resistive impedance decrease with the increase of plasma layer thickness, Jeffrey fluid parameter, Darcy number and Darcy slip parameter, and increase with the rise of hematocrit. The results in the case of variable core viscosity and constant core viscosity are compared to investigate the impact of variable core viscosity in managing the flow of blood.
\end{abstract}

Keywords. Jeffrey fluid; porous wall; hematocrit; Brinkman layer; Darcy region; variable core fluid viscosity.

\section{Introduction}

In the modern days, cardiovascular diseases are the leading cause of death among men and women in the world, and the heart diseases mainly occur due to coronary artery diseases [1]. Coronary artery disease is the shrink or occlusion of the coronary arteries, usually caused by stenosis. Stenosis ("solidification" of arteries) is the accumulation of cholesterol and plaques on the inner walls of the arteries. The stenosis can hinder the flow of blood to the heart by physically choking the blood vessel and function. Due to the insufficient blood supply, the heart suffers from the lack of oxygen and the crucial nutrients it needs to work. If the blood supply to the heart is cut-off altogether, or if the vitality demands of the heart become much higher than its blood supply, a heart attack or stroke may occur [2].

To understand the effect of stenosis on the blood flow characteristics, namely velocity profile, wall shear stress and resistive impedance, many researchers [3-5] have discussed the experimental and theoretical studies on blood flow through the arteries with mild stenosis, which is very helpful for the diagnosis of cardiovascular diseases and in the design of medical devices that are used in diagnosing

*For correspondence cardiovascular diseases. Young [6] and Mishra et al [7] have analysed the characteristics of the blood flow through the arterial stenosis by treating the blood as a Newtonian fluid. However, it is well known that blood behaves as a non-Newtonian fluid when it flows through small diameter arteries at low shear rates [8-10]. On account of this, numerous authors [11-13] studied the blood flow characteristics in mild stenosed arteries by assuming blood as a non-Newtonian fluid.

Blood flow through stenosed arteries has been represented by Newtonian or non-Newtonian, single-layered models by the investigators in the literature. It is well known that blood may be described by a single-layered model in large blood vessels; however, blood flowing through narrow arteries is considered to require a doublelayered model. Bugliarello and Hyden [14], Bugliarello and Sevilla [15] and Cokelet [16] have experimentally shown that when blood flows through small blood vessels, there exists a cell-free plasma layer (Newtonian viscous fluid) near the wall. In the light of their experiments, it is more appropriate to represent the blood flow through narrow arteries by a two-layered model instead of the one-layered model. Shukla et al [17], Chaturani and Ponnalagarsamy [18], and Priyadharshini and Ponalagusamy [19] have discussed two-layered models in which the peripheral layer is 
a Newtonian fluid, and the core region is a non-Newtonian fluid (blood). Nallapu and Radhakrishnamacharya [20] have dealt with the problem of two-layered model of blood flow in small diameter tubes, where the core region consists of a Jeffrey fluid with constant viscosity and Newtonian fluid in the peripheral zone of plasma. They obtained analytical expressions for velocity, effective viscosity, core hematocrit and mean hematocrit. Later Sharma et al [21] extended this work for a stenosed narrow tube. Ponalagusamy [22] studied a two-layered model of the steady flow of blood through stenosed tubes where the core region is occupied by a particle-fluid suspension (Jeffrey fluid) and the peripheral plasma layer is a Newtonian fluid. The study revealed that the shear stress at the wall and flow impedance reduce with the rise in Jeffrey fluid parameter (ratio of retardation time to relaxation time).

Most of the models on the blood flow deal with a constant core viscosity. Bugliarello and Sevilla [15] experimentally observed that most of the blood cells are concentrated near the central region (axis) of the blood vessel and less towards the blood vessel wall, a cell-free zone, and thus the non-homogeneity of blood should be considered while studying the blood flow through small diameter arteries. Hence the core viscosity of blood is to be a function of hematocrit and radial distance, and it varies in such a way that it is higher near the central core region and reduces when one moves towards the blood vessel wall and becomes a constant (Newtonian fluid viscosity) in the peripheral layer of plasma. There are a few articles that analyse the effects of variable core fluid viscosity on the blood flow through the stenosed arteries. Ponalagusamy and Tamil Selvi [23] analysed the two-fluid model of blood flow in stenotic arteries in which the fluid in both core and plasma layer is considered to obey the law of Newtonian fluid and considering the core viscosity of blood as a radial coordinate dependent. Priyadharshini and Ponalagusamy [19] examined a two-layered model of blood flow in an unsteady state where blood in the central core region is assumed to be a non-Newtonian (Jeffrey) fluid and a Newtonian fluid in the peripheral plasma layer by taking core viscosity as variable and applying a radially variable external magnetic field. Ponalagusamy [24] developed a two-layered model concerning measurable flow variables for flow of blood in a stenosed artery and derived formulas for computing axially variable plasma layer thickness, variable core fluid viscosity and axially variable slip velocity.

Porous medium is a material that consists of a solid frame with interconnected pores. Permeability is another important character of the porous medium, which is a measure of the ability of a porous material to allow fluids to pass through it. Fluid flow in a porous medium has been studied by employing Darcy's law [25]. Brinkman [26] extended Darcy's law using the boundary condition. Raj and Sen [27] analysed the flow through compliant microchannels in which one of the channel walls is a thin compliant polymer membrane. The pressure-flow rate characteristics of a shear thinning non-Newtonian fluid ( $0.1 \%$ polyethylene oxide solution) in the compliant and conventional (rigid) microchannels are experimentally measured and compared. They found that in both channels (compliant and conventional), initially the pressure drop increases due to flow rate but then decreases with flow rate due to the shear thinning effects. The entry and passage behaviour of biological cells (HeLa and MDA-MB-231) in a constricted compliant microchannel has been investigated by Raj and Sen [28]. Raj et al [29] investigated the droplet generation behaviour of a microfluidic droplet generator with a controllably deformable membrane wall using experiments and an analytical model. Nallapu et al [30] discussed the two-fluid model of blood flow through uniform circular tubes in a porous medium by assuming the core region as a Jeffrey fluid and plasma layer as a Newtonian fluid. It is observed by Ponalagusamy [31] that the wall shear stress and resistive impedance are decreased with the rise of Darcy number and the introduction of a plasma layer near the arterial wall. In the studies mentioned earlier, the blood flow pertains to one-layer or two-layer models.

It is understood that the arterial wall is composed of three layers, namely, tunica intima, tunica media and tunica adventitia [32]. The tunica intima is the innermost layer of a blood vessel, comprising a single continuous layer of endothelium supported by a sub-endothelial layer. The endothelium forms a barrier between the circulating blood and surrounding tissues and also contributes to the regulation of coagulation, inflammation and vessel tone. The tunica intima is surrounded by the tunica media, which comprises smooth muscle cells, and elastic and connective tissues arranged circularly around the blood vessel. The outermost layer of a blood vessel is the tunica externa or tunica adventitia, composed entirely of connective fibres and bounded by an external elastic lamina, which functions to anchor vessels with surrounding tissues. The integrity of the endothelium and its overlying tissues is essential for normal blood flow in an artery. The reason is that atherosclerosis begins in the wall of the artery with an early abnormality in the lining of the arterial wall called the endothelium. Haemodynamics interactions between the endothelium and the blood sustain a healthy situation that prevents the formation of clots and stenosis. Damage or disturbance in the endothelium and its surrounding tissues due to flow conditions triggers a localized release of chemical mediators and cellular responses that lead to thrombus formation, overproduction of endothelins, increase in blood pressure, deposition of atherosclerosis and haemostasis [33]. Because of this, the seepage of fluid (blood) into these layers is physiologically pertinent to be investigated. Goharzadeh et al [34] and Hill and Straughan [35] considered the three layers of the channel such that the porous wall consists of a thin transition layer (Brinkman) overlying a Darcy region. 
Goharzadeh et al [34] experimentally analysed the transition layer at a fluid-porous interface, and they measured the thickness of the transition layer (Brinkman layer thickness). Hill and Straughan [35] studied a three-layer model in which fluid overlies a porous medium (Brinkman and Darcy layers) in the absence of the plasma layer near the Brinkman layer. They investigated the instability of Poiseuille flow in the three-layer model where the porous medium is soaked with the same fluid. They found that the critical parameters that influence the stability features of the system are the ratio of the depth of the fluid layer to that of porous layers, and also the Brinkman layer thickness. Boodoo et al [36] introduced a theoretical study of a twofluid model of blood flow through a uniform artery considering a core region with micro-polar fluid and Newtonian fluid in peripheral plasma region and the wall of the capillary with the porous medium comprising a thin transition layer (Brinkman) surrounded by a Darcy region. The study showed that the system is sensitive to variations in micropolar coupling number and also found that higher values of hydraulic resistivity develop slower velocities of the micropolar and Newtonian fluids. It is pertinent to pinpoint here that constant core viscosity is taken in [35-37], which is not valid because radial migration of blood cells has been experimentally observed for the case of blood flow in small arteries and capillaries. Furthermore, they have not discussed the physiologically significant axial variation of flow resistance for the parameters involved in their analysis. It is well known that the fact that distribution of wall shear stress and flow resistance is very much altered by variable core viscosity should be considered while analysing the flow of blood. In the literature, no effort has, at least to the author's knowledge, been made to develop fourlayered model for the flow of Jeffrey fluid (blood) in stenosed arteries by taking in to account variable core fluid viscosity. Keeping this in mind, a modest effort has been made in the present investigation to study the four-layered model of blood flow through an artery with stenosis and the core region as a non-Newtonian (Jeffrey) fluid model whose viscosity is radial and axial coordinate dependent and peripheral plasma region with a Newtonian fluid. The porous wall of the stenosed artery is composed of a transition Brinkman layer (tunica intima) accompanied by a Darcy region (tunica media and tunica adventitia).

\section{Mathematical modelling}

Let us consider a four-layered model for blood flow through a stenosed artery, which consists of a core region, plasma layer and the two-layer porous wall. Consider an axisymmetric, laminar, steady, fully developed and incompressible blood flow in the axial direction $(\bar{z})$ through the artery with axially non-symmetric but radially symmetric mild stenosis. The two-layer wall of the stenosed artery with porous medium consists of an inner layer as a thin transition (Brinkman) layer and the outer layer as a Darcy layer filled with the Newtonian fluid. It is assumed that the rheology of blood in the central core region of suspension of all the erythrocytes is that of a non-Newtonian (Jeffrey) fluid and a peripheral layer of plasma as a Newtonian fluid. We take $(\bar{r}, \bar{\theta}, \bar{z})$ as cylindrical coordinates such that the axis of the artery is taken along $\bar{z}$-axis, $\bar{r}$ is taken along the radius of the artery and $\bar{\theta}$ represents the circumferential direction. The geometry of the flow in a stenosed artery is shown in figure 1 and described [22] as

$$
\bar{R}(\bar{z})=\left\{\begin{array}{lc}
\bar{R}_{0}\left[1-\bar{A}\left\{\bar{L}_{0}^{n-1}(\bar{z}-\bar{d})-(\bar{z}-\bar{d})^{n}\right\}\right], & \bar{d} \leq \bar{z}<\bar{d}+\bar{L}_{0} \\
\bar{R}_{0}, & \text { otherwise }
\end{array}\right.
$$

where $\bar{R}_{0}$ is the radius of the normal artery, $\bar{L}_{0}$ is the length of the constriction, $\bar{d}$ indicates its location and $n(\geq 2)$ is shape parameter, determining the shape of the stenosis. The parameter $\bar{A}$ is given by $\bar{A}=\frac{\bar{\delta}_{s}}{\bar{R}_{0} \bar{L}_{0}^{n}}\left(\frac{n^{n /(n-1)}}{n-1}\right)$, where $\bar{\delta}_{s}$ is the maximum depth of the constriction located at $\bar{z}=$ $\bar{d}+\frac{\bar{L}_{0}}{n^{1 /(n-1)}}$, such that $\frac{\bar{\delta}_{s}}{\bar{R}_{0}}<<1$. Let $\bar{R}_{C}(\bar{z}), \bar{R}_{P}(\bar{z}), \bar{R}_{B}(\bar{z})$ and $\bar{R}_{D}(\bar{z})$ represent the radius of the blood vessel in the stenosed region for core, plasma, Brinkman and Darcy region, respectively. Also let $\bar{h}_{P}, \bar{h}_{B}$ and $\bar{h}_{D}$ denote the thickness of the plasma, Brinkman and Darcy layers, respectively, such that $\bar{h}_{B}=\bar{h}_{D} / 9$ [38]; we assume that $\bar{p}_{C}, \bar{p}_{P}, \bar{p}_{B}$ and $\bar{p}_{D}$ denote the pressures in the four regions, respectively. Since the flow is axisymmetric, all the flow variables are independent of circumferential coordinate $\bar{\theta}$. Hence the velocity vector is given by $\bar{V}=\left(\bar{v}_{i}, 0, \bar{u}_{i}\right), i=$ $C, P, B$ and $D$, where $\bar{u}_{i}$ is the axial velocity and $\bar{v}_{i}$ is the radial velocity in these four regions.

Based on the work in $[1,22,35,36]$ for a low-Reynoldsnumber flow through an artery with mild stenosis, the radial velocity in all the four regions is negligibly small and can be neglected. Because of this, the equation of continuity and the momentum equations in all the four regions are described as follows.

Region I: Non-Newtonian (Jeffrey fluid) region $\left[0 \leq \bar{r} \leq \bar{R}_{C}\right]$

$$
\frac{\partial \bar{p}_{C}}{\partial \bar{z}}-\frac{1}{\bar{r}} \frac{\partial}{\partial \bar{r}}\left(\bar{r} \bar{S}_{\bar{r} \bar{z}}\right)=0
$$

where $\bar{S}_{\bar{r} \bar{z}}$ is shear stress component in the $\bar{r} \bar{z}$-plane and is given by

$$
\bar{S}_{\bar{r} \bar{z}}=\frac{\bar{\mu}_{C}(\bar{r})}{1+\lambda_{1}} \frac{\partial \bar{u}_{C}}{\partial \bar{r}} .
$$




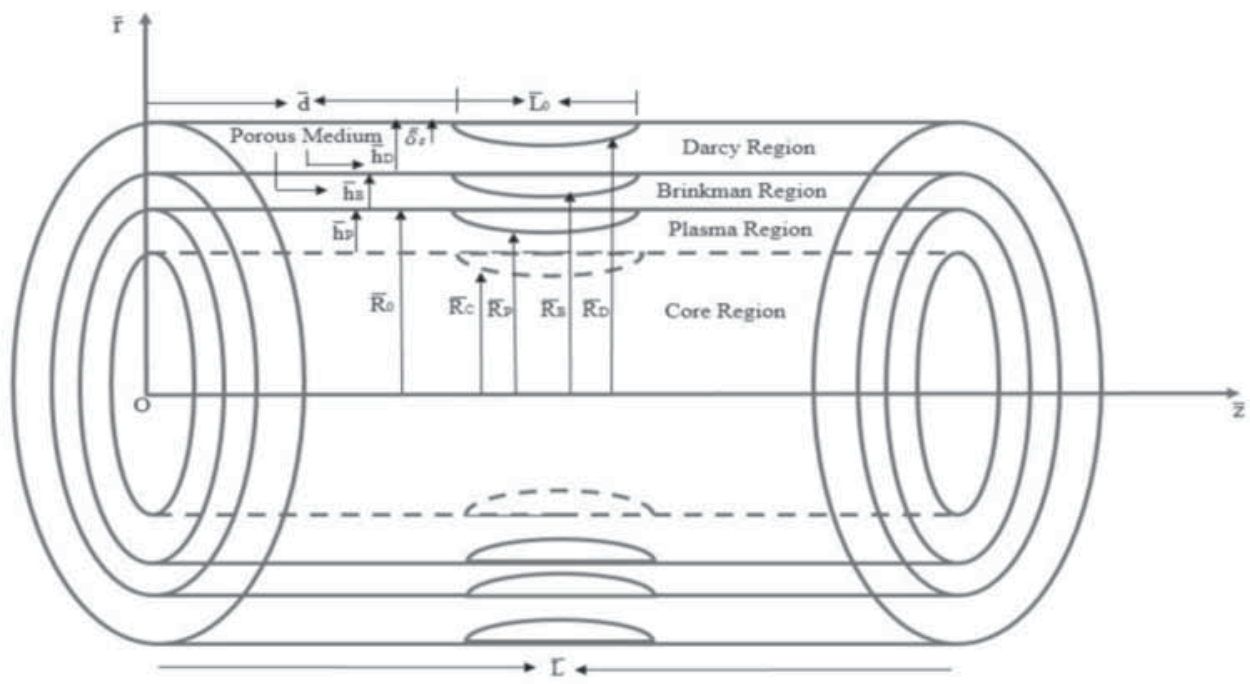

Figure 1. Schematic diagram of arterial stenosis with various regions.

Here $\bar{\mu}_{C}(\bar{r})=\bar{\mu}_{N}+\bar{\mu}_{N} \eta h_{m}\left[\left(\frac{\bar{R}_{C}}{\bar{R}_{0}}\right)^{2}-\left(\frac{\bar{r}}{\bar{R}_{0}}\right)^{2}\right], \bar{\mu}_{C}(\bar{r})$ is the viscosity of Jeffrey fluid, $\bar{\mu}_{N}$ is the viscosity of Newtonian fluid, $\eta$ is constant and $h_{m}$ is the concentration of red blood cells (volume fraction density of cells or hematocrit).

Region II: Plasma (Newtonian fluid) region $\left[\bar{R}_{C} \leq \bar{r} \leq \bar{R}_{P}\right]$

$$
\frac{\partial \bar{p}_{P}}{\partial \bar{z}}-\frac{\bar{\mu}_{N}}{\bar{r}} \frac{\partial}{\partial \bar{r}}\left(\bar{r} \frac{\partial \bar{u}_{P}}{\partial \bar{r}}\right)=0
$$

Region III: Brinkman porous region $\left[\bar{R}_{P} \leq \bar{r} \leq \bar{R}_{B}\right]$

$$
\frac{\partial \bar{p}_{B}}{\partial \bar{z}}-\frac{\bar{\mu}_{e}}{\bar{r}} \frac{\partial}{\partial \bar{r}}\left(\bar{r} \frac{\partial \bar{u}_{B}}{\partial \bar{r}}\right)+\frac{\bar{\mu}_{N}}{\bar{k}} \bar{u}_{B}=0 .
$$

Region IV: Darcy porous region $\left[\bar{R}_{B} \leq \bar{r} \leq \bar{R}_{D}\right]$

$$
\frac{\partial \bar{p}_{D}}{\partial \bar{z}}+\frac{\bar{\mu}_{N}}{\bar{k}} \bar{u}_{D}=0
$$

where $\bar{\mu}_{e}$ is the effective viscosity of transition layer and $\lambda_{1}$ is the ratio of relaxation to retardation times and $\bar{k}$ is the permeability constant.

Let us introduce the following non-dimensional variables:

$$
\begin{aligned}
& r=\frac{\bar{r}}{\bar{R}_{0}}, z=\frac{\bar{z}}{\bar{R}_{0}}, d=\frac{\bar{d}}{\bar{R}_{0}}, L_{0}=\frac{\bar{L}_{0}}{\bar{R}_{0}}, \delta_{s}=\frac{\bar{\delta}_{s}}{\bar{R}_{0}}, R(z)=\frac{\bar{R}(\bar{z})}{\bar{R}_{0}}, \\
& R_{i}(z)=\frac{\bar{R}_{i}(\bar{z})}{\bar{R}_{0}}, u_{i}=\frac{\bar{u}_{i}}{\bar{u}_{0}}, p_{i}=\frac{\bar{R}_{0} \bar{p}_{i}}{\bar{u}_{0} \bar{\mu}_{N}}(i=C, P, B, D), \\
& \alpha_{1}(r)=\frac{\bar{\mu}_{C}(\bar{r})}{\bar{\mu}_{N}}, \alpha_{2}=\frac{\bar{\mu}_{e}}{\bar{\mu}_{N}}, D_{a}=\frac{\bar{k}}{\bar{R}_{0}^{2}}, S_{r z}=\frac{\bar{R}_{0} \bar{S}_{\bar{r} \bar{z}}}{\bar{u}_{0} \bar{\mu}_{N}}
\end{aligned}
$$

where $\bar{u}_{0}$ is the average velocity over the cross section of the uniform artery of radius $\bar{R}_{0}, \alpha_{2}$ is the non-dimensional effective viscosity and $D_{a}$ is the porosity parameter (Darcy number). Using these non-dimensional variables, the simplified governing equations in these four regions are given here.

Region I: Non-Newtonian (Jeffrey fluid) region $\left[0 \leq r \leq R_{C}\right]$

$$
\frac{d p_{C}}{d z}-\frac{1}{r} \frac{d}{d r}\left(r S_{r z}\right)=0
$$

where the rheological equation for Jeffrey fluid is given by

$$
S_{r z}=\left[\frac{\alpha_{1}(r)}{1+\lambda_{1}}\right] \frac{d u_{C}}{d r}
$$

Region II: Plasma (Newtonian fluid) region $\left[R_{C} \leq r \leq R_{P}\right]$

$$
\frac{d p_{P}}{d z}-\frac{1}{r} \frac{d}{d r}\left(r \frac{d u_{P}}{d r}\right)=0
$$

Region III: Brinkman porous region $\left[R_{p} \leq r \leq R_{B}\right]$

$$
\frac{d p_{B}}{d z}-\frac{\alpha_{2}}{r} \frac{d}{d r}\left(r \frac{d u_{B}}{d r}\right)+\frac{1}{D_{a}} u_{B}=0
$$

Region IV: Darcy porous region $\left[R_{B} \leq r \leq R_{D}\right]$

$$
\frac{d p_{D}}{d z}+\frac{1}{D_{a}} u_{D}=0
$$

where $\alpha_{1}(r)$ is the variable core fluid viscosity and is taken as

$$
\alpha_{1}(r)=1+\eta h_{m}\left(R_{C}^{2}-r^{2}\right)
$$


$\eta$ is a constant and $h_{m}$ is the haematocrit (packed red blood cells).

The corresponding boundary conditions are given as follows:

$$
\begin{gathered}
\text { (i) at } r=0, \quad S_{r z} \text { is finite } \\
\text { (ii) } S_{C}=S_{P} \text { at } r=R_{C} \\
\text { (iii) } u_{C}=u_{P} \text { at } r=R_{C} \\
\text { (iv) } u_{P}=u_{B} \text { at } r=R_{P} \\
\text { (v) } \frac{1}{\phi}\left(\frac{d u_{B}}{d r}\right)-\left(\frac{d u_{P}}{d r}\right)=\frac{\beta}{\sqrt{D_{a}}} u_{B} \text { at } r=R_{P}
\end{gathered}
$$

where $\phi$ is the porosity and $\beta$ is the stress jump parameter [39];

$$
\text { (vi) } \frac{d u_{B}}{d r}=\frac{\alpha}{\sqrt{D_{a}}}\left(u_{B}-u_{D}\right) \text { at } r=R_{B}
$$

where $\alpha$ is the Darcy slip parameter [40].

\section{Method of solution}

Let us assume that the pressure gradient in all the four regions be the same, say $P_{0}(z)$.

Substituting $-\frac{d p_{C}}{d z}=P_{0}$ in Eq. (8) and integrating, we can obtain

$$
S_{r z}=-\frac{P_{0} r}{2}+\frac{C_{1}}{r} .
$$

The solutions of Eqs. (9)-(12) using Eq. (13) can be obtained as

$$
\begin{gathered}
u_{C}=\frac{P_{0}\left(1+\lambda_{1}\right)}{4 \eta h_{m}} \ln \left[1+\eta h_{m}\left(R_{C}^{2}-r^{2}\right)\right]+C_{2} \\
u_{P}=-\frac{r^{2} P_{0}}{4}+\ln (r) C_{3}+C_{4} \\
u_{B}=I_{0}(\sigma r) C_{5}+K_{0}(\sigma r) C_{6}+P_{0} D_{a} \\
u_{D}=P_{0} D_{a}
\end{gathered}
$$

where $\sigma=\frac{1}{\sqrt{\alpha_{2} D_{a}}}, I_{0}$ and $K_{0}$ are modified Bessel functions and $C_{1}-C_{6}$ are arbitrary constants to be determined.

Applying boundary condition (i), we get

$$
C_{1}=0
$$

Similarly, applying boundary condition (ii), we get

$$
C_{3}=0
$$

Applying boundary conditions (iii)-(vi), one can obtain

$$
\begin{gathered}
-C_{2}+C_{4}=\frac{P_{0} R_{C}^{2}}{4}, \\
C_{4}-I_{0}\left(\sigma R_{P}\right) C_{5}-K_{0}\left(\sigma R_{P}\right) C_{6}=\frac{P_{0}\left(R_{P}^{2}+4 D_{a}\right)}{4}, \\
{\left[\phi \beta I_{0}\left(\sigma R_{P}\right)-\sigma \sqrt{D_{a}} I_{1}\left(\sigma R_{P}\right)\right] C_{5}} \\
+\left[\sigma \sqrt{D_{a}} K_{1}\left(\sigma R_{P}\right)+\phi \beta K_{0}\left(\sigma R_{P}\right)\right] C_{6} \\
=\frac{\phi P_{0}\left(R_{P} \sqrt{D_{a}}-2 \beta D_{a}\right)}{2} \\
{\left[\sigma \sqrt{D_{a}} I_{1}\left(\sigma R_{B}\right)-\alpha I_{0}\left(\sigma R_{B}\right)\right] C_{5}} \\
-\left[\sigma \sqrt{D_{a}} K_{1}\left(\sigma R_{B}\right)+\alpha K_{0}\left(\sigma R_{B}\right)\right] C_{6}=0 .
\end{gathered}
$$

Solving the system of Eqs. (27)-(30) by employing the MATLAB software, we can obtain the expressions for the arbitrary constants $C_{2}, C_{4}, C_{5}$ and $C_{6}$, which are given in Appendix I.

The total volumetric flow rate is given by

$$
Q=Q_{C}+Q_{P}+Q_{B}+Q_{D}
$$

where $Q_{C}, Q_{P}, Q_{B}$ and $Q_{D}$ are the volumetric flow rates in core, plasma, Brinkman and Darcy regions, respectively.

The volumetric flow rate for core region is defined as

$$
Q_{C}=\int_{0}^{R_{C}} r u_{C} d r
$$

Substituting Eq. (21) in (32) and integrating, we get

$$
\begin{aligned}
Q_{C}= & \frac{P_{0}\left(1+\lambda_{1}\right)}{8 \eta h_{m}}\left[\left(\frac{1+\eta h_{m} R_{C}^{2}}{\eta h_{m}}\right) \ln \left(1+\eta h_{m} R_{C}^{2}\right)-R_{C}^{2}\right] \\
& +\frac{C_{2}}{2} R_{C}^{2} .
\end{aligned}
$$

Similarly, the expressions for $Q_{P}, Q_{B}$ and $Q_{D}$ for the other three regions can be obtained as

$$
\begin{aligned}
Q_{P}= & -\frac{P_{0}}{16}\left(R_{P}^{4}-R_{C}^{4}\right)+\frac{C_{4}}{2}\left(R_{P}^{2}-R_{C}^{2}\right), \\
Q_{B}= & \frac{C_{5}}{\sigma}\left[R_{B} I_{1}\left(\sigma R_{B}\right)-R_{P} I_{1}\left(\sigma R_{P}\right)\right] \\
& -\frac{C_{6}}{\sigma}\left[R_{B} K_{1}\left(\sigma R_{B}\right)-R_{P} K_{1}\left(\sigma R_{P}\right)\right] \\
& +\frac{P_{0} D_{a}}{2}\left(R_{B}^{2}-R_{P}^{2}\right) \\
& Q_{D}=\frac{P_{0} D_{a}}{2}\left(R_{D}^{2}-R_{B}^{2}\right) .
\end{aligned}
$$

Using Eqs. (33)-(36) in Eq. (31), the total volume flow rate is obtained as 


$$
\begin{aligned}
Q= & \frac{P_{0}\left(1+\lambda_{1}\right)}{8\left(\eta h_{m}\right)^{2}}\left[\left(1+\eta h_{m} R_{C}^{2}\right) \ln \left(1+\eta h_{m} R_{C}^{2}\right)-\eta h_{m} R_{C}^{2}\right] \\
& +\frac{C_{2}}{2} R_{C}^{2}-\frac{P_{0}}{16}\left(R_{P}^{4}-R_{C}^{4}\right)+\frac{C_{4}}{2}\left(R_{P}^{2}-R_{C}^{2}\right) \\
& +\frac{C_{5}}{\sigma}\left[R_{B} I_{1}\left(\sigma R_{B}\right)-R_{P} I_{1}\left(\sigma R_{P}\right)\right] \\
& -\frac{C_{6}}{\sigma}\left[R_{B} K_{1}\left(\sigma R_{B}\right)-R_{P} K_{1}\left(\sigma R_{P}\right)\right] \\
& +\frac{P_{0} D_{a}}{2}\left(R_{D}^{2}-R_{P}^{2}\right) .
\end{aligned}
$$

The values of the pressure gradient $P_{0}$ can be computed for various values of the parameters involved in the present work by taking total flow rate $Q$ to be unity. MATLAB package is used to compute the values of the pressure gradient.

The shear stress at the wall of the arterial stenosis (wall shear stress) is defined as

$$
\tau_{w}=\left(-\frac{d u_{P}}{d r}\right)_{r=R_{P}}+\left(-\frac{d u_{B}}{d r}\right)_{r=R_{B}}-\left(-\frac{d u_{B}}{d r}\right)_{r=R_{P}} .
$$

Using the values of $u_{P}$ and $u_{B}$ from Eqs. (22) and (23) in Eq. (38), the wall shear stress $\tau_{w}$ may be expressed as

$$
\begin{aligned}
\tau_{w}= & \frac{P_{0} R_{P}}{2}+\sigma C_{6}\left(K_{1}\left(\sigma R_{B}\right)-K_{1}\left(\sigma R_{P}\right)\right) \\
& -\sigma C_{5}\left(I_{1}\left(\sigma R_{B}\right)-I_{1}\left(\sigma R_{P}\right)\right) .
\end{aligned}
$$

The resistive impedance $\lambda$ is calculated as

$$
\lambda=\int_{0}^{L} \frac{P_{0}(z)}{Q} d z
$$

where $L$ is the length of the blood vessel. By numerical integration on Eq. (40), we can compute numerical values of flow impedance for several values of the parameters involved in the present investigation.

\section{Discussion}

The flow of blood is pigeonholed by a four-layered model comprising a cell-rich core region of suspension of all the erythrocytes described to be a fluid suspension, a peripheral layer of plasma free from cells, Brinkman layer and Darcy region. A modest effort is made to understand the simultaneous effects of non-Newtonian behaviour of blood, plasma layer thickness, radially variable core viscosity, Darcy number, the porosity, stress jump parameter, Darcy slip parameter and thickness of Brinkman and Darcy regions on physiologically pivotal flow variables such as velocity profile, skin friction (WSS) and flow impedance. The value of the pressure gradient (a positive root) is numerically computed for various values of the parameters involved in the present work using the subroutine "root $(f(y), y, a, b)$ " of the MATLAB package. For computational purpose, the values of the parameters involved in the present analysis are tabulated in table 1.

It is verified that the present flow model reduces to onelayered model developed by Young [1] for the flow of Newtonian fluid with constant viscosity through the stenosed artery by setting $\lambda_{1}=0, h_{P}=0, h_{D}=0$. Further, it is reduced to two-layered models studied by Nallapu and Radhakrishnamacharya [20] for $\lambda_{1}=1, h_{P}=0.05, h_{D}=0$, $\delta_{s}=0$ and Sharma et al [21] for $\lambda_{1}=1, h_{P}=0.05, h_{D}=0$ for the flow of non-Newtonian (Jeffrey) fluid in the core region and Newtonian fluid in the peripheral plasma layer when blood flows through the rigid uniform tube and rigid arterial stenosis, respectively, by assuming constant core fluid viscosity with no slip condition at the wall.

Figure 2 compares the presently computed velocity profiles with those obtained by other investigators $[1,20,21]$ under several flow conditions. It is noted that the velocity profile obtained from the present study is in a good agreement with the respective mathematical model developed by Young [1], Nallapu and Radhakrishnamacharya [20] and Sharma et al [21]. The influence of variable viscosity on the velocity of blood in comparison with constant viscosity models of blood flow through small blood vessels has also been illustrated in figure 2 .

Figure 3 illustrates the radial distribution of axial velocity with the Jeffrey fluid parameter $\left(\lambda_{1}\right)$ and thickness of plasma layer $\left(h_{P}\right)$. Figure 3 reveals that the axial velocity tends to increase with the rise in $\lambda_{1}$ and $h_{P}$. For a Jeffrey fluid, the increase of plasma layer thickness near the Brinkman layer increases the fluid velocity around the axis of the stenosed artery, but it decreases the velocity as one moves towards the Brinkman region. Moreover, this phenomenon becomes insignificant for the flow of a Newtonian

Table 1. Governing parameters and their ranges.

\begin{tabular}{lcc}
\hline Parameters & $\begin{array}{c}\text { Symbol } \\
\text { (dimensionless) }\end{array}$ & $\begin{array}{c}\text { Values } \\
{[1,22,38]}\end{array}$ \\
\hline Length of stenosis & $L_{0}$ & 1 \\
Location of stenosis & $d$ & 4 \\
Maximum stenotic height & $\delta_{s}$ & $0-0.2$ \\
Shape of stenosis & $n$ & $2-6$ \\
Thickness of plasma layer & $h_{P}$ & $0-0.1$ \\
Thickness of Darcy region & $h_{D}$ & $0.2-1.8$ \\
Jeffrey fluid parameter & $\lambda_{1}$ & $0-3$ \\
Haematocrit & $h_{m}$ & $0.2-0.5$ \\
Effective viscosity & $\alpha_{2}$ & 1.1 \\
Darcy slip parameter & $\alpha$ & $0.01-0.1$ \\
Stress jump parameter & $\beta$ & 0.1 \\
Porosity & $\phi$ & 0.5 \\
Constant parameter in core & $\eta$ & $8-10$ \\
$\quad$ fluid viscosity & & \\
Darcy number & $D_{a}$ & $0.01-0.3$ \\
\hline
\end{tabular}




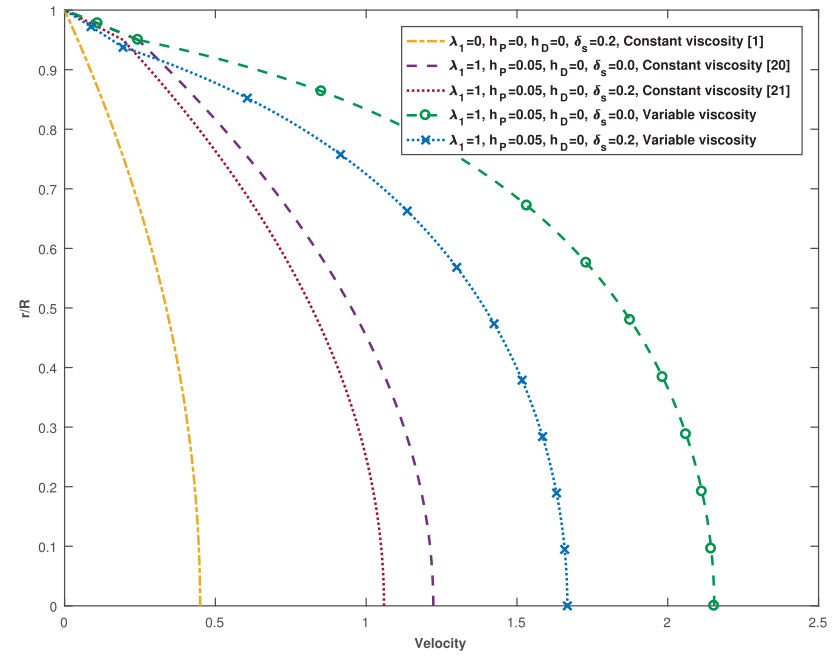

Figure 2. Radial distribution of velocity of blood with onelayered and two-layered models for flow of Newtonian and Jeffrey fluids in a rigid arterial stenosis.

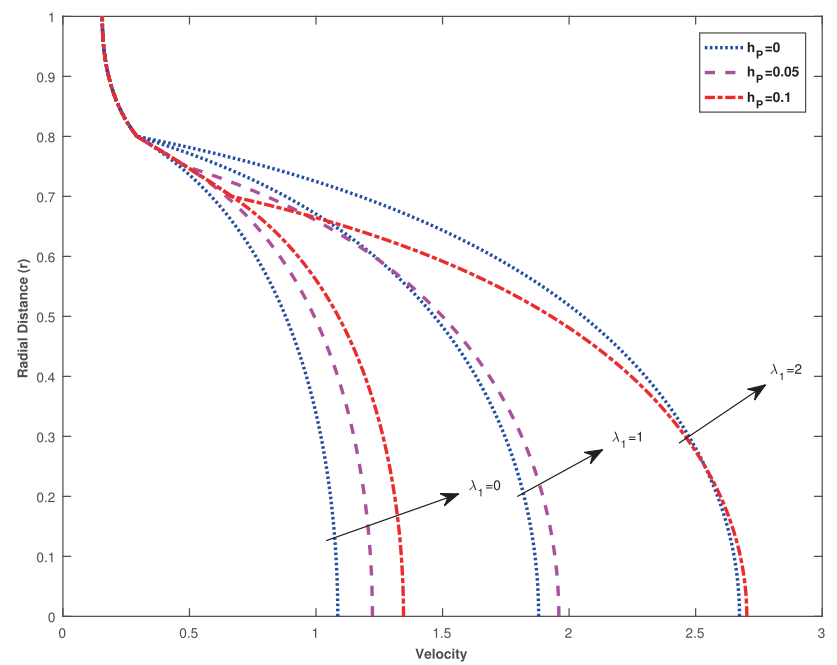

Figure 3. Radial variation of velocity of blood with plasma layer thickness $\left(h_{P}\right)$ and Jeffrey fluid parameter $\left(\lambda_{1}\right)$ at the mid-point of stenosis.

fluid. The results of distribution of velocity versus the radial distance $(r)$ against the values of haematocrit $\left(h_{m}\right)$ is shown in figure 4. We see that an increase in the haematocrit (concentration of cells in the core region) leads to a decrease in the velocity and the rate of decrease in the velocity with the increase in the radial distance has been found to be higher for a lower value of $h_{m}$.

The effects of thickness of Darcy region $\left(h_{D}\right)$, Darcy number $\left(D_{a}\right)$ and Darcy slip parameter $(\alpha)$ on the radial variation of velocity are depicted in figures 5 and 6 . At this juncture, we note that since the ratio of the thickness of Brinkman layer to that of Darcy region is considered to be $1 / 9$ [38], the value of $h_{B}$ is changed in accordance with the

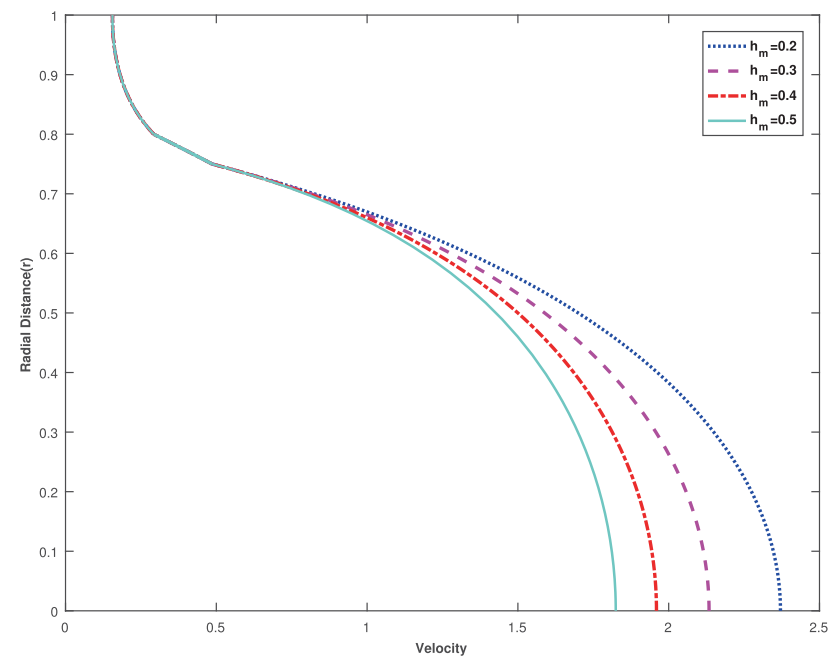

Figure 4. Variation of velocity of blood with haematocrit $\left(h_{m}\right)$ at the mid-point of stenosis.

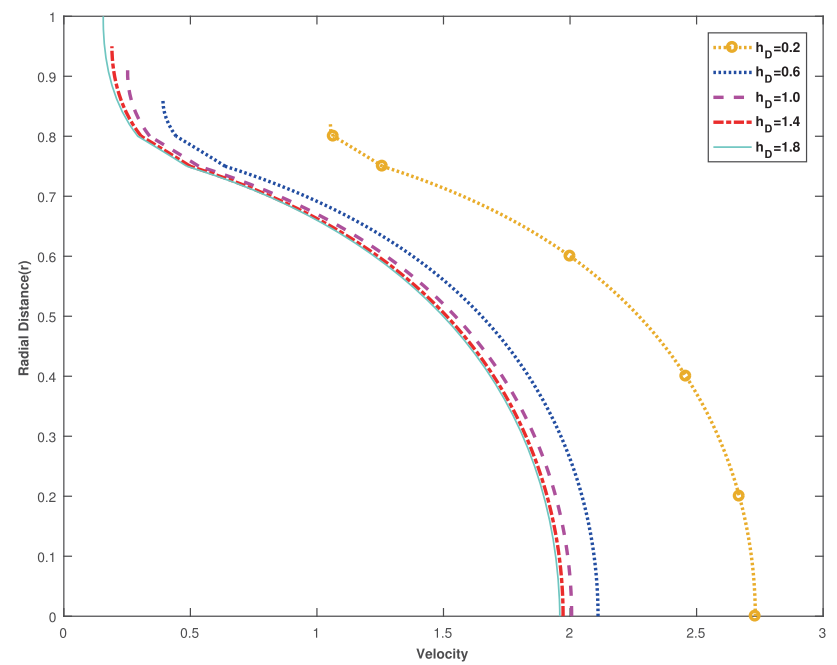

Figure 5. Variation of velocity of blood with Darcy layer thickness $\left(h_{D}\right)$ at the mid-point of stenosis.

change in the value of a thickness of Darcy region $\left(h_{D}\right)$. We observe from figure 5 that the velocity at the maximum depth of the stenotic region decreases as the value of $h_{D}$ increases. For a lower value of thickness of Darcy region $\left(h_{D}\right)$, the percentage decrease in the velocity is found to be significant, and this becomes less significant for a higher value of thickness of Darcy region $\left(h_{D}\right)$. One notices from figure 6 that for any given set of other parameters, the velocity is found to increase with the increase in Darcy number and Darcy slip parameter. We infer from the result that the velocity in both the core and peripheral plasma regions is higher in the case of the flow of blood through a rigid arterial stenosis. Another notable observation is that Darcy slip parameter is a weak parameter in comparison with Darcy number in the sense that the former parameter 


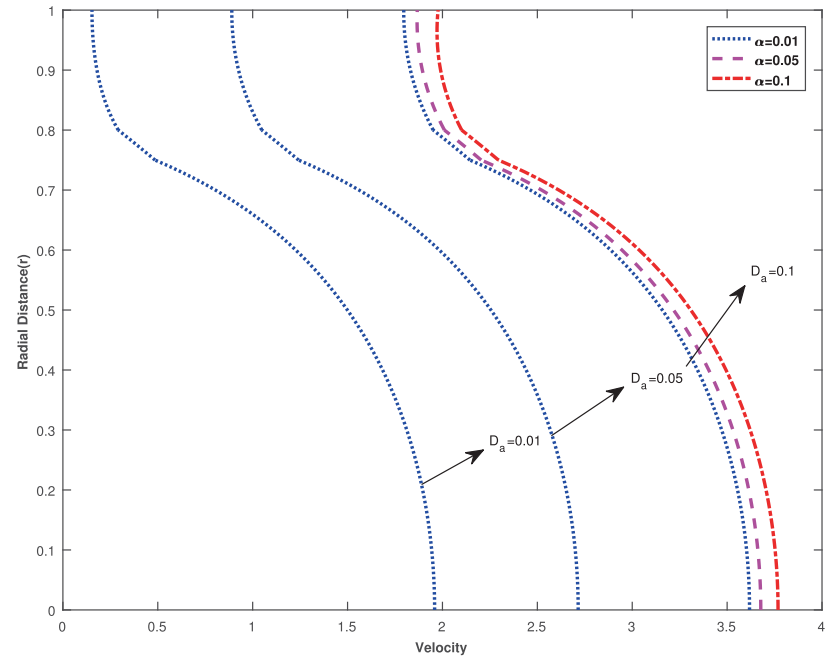

Figure 6. Variation of velocity of blood with Darcy number $\left(D_{a}\right)$ and Darcy slip parameter $(\alpha)$ at the mid-point of stenosis.

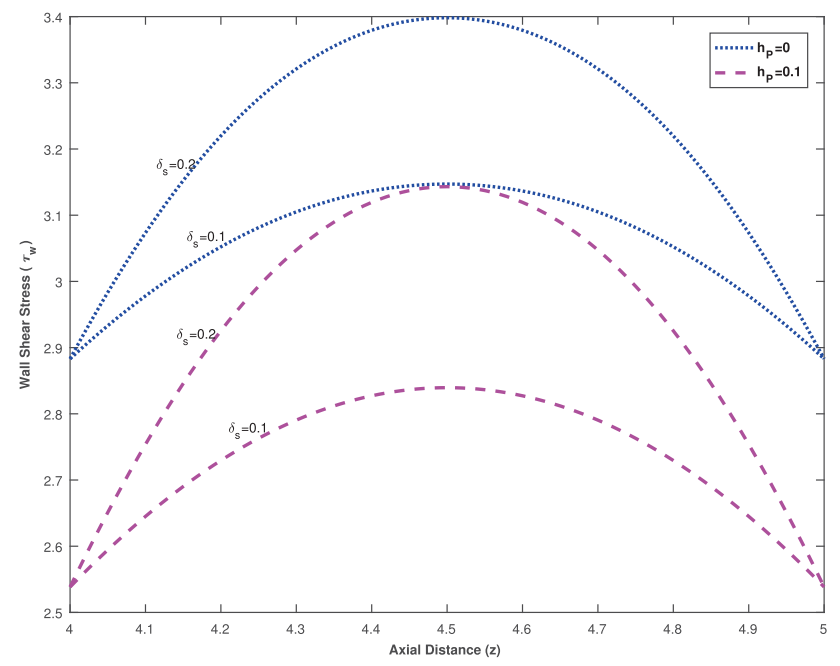

Figure 7. Axial variation of wall shear stress $\left(\tau_{w}\right)$ with plasma layer thickness $\left(h_{P}\right)$ for a Newtonian fluid $\left(\lambda_{1}=0\right)$.

brings a smaller increment in the magnitude of the axial velocity for the values of the parameters considered in the present investigation.

Evaluating the values of shear stress at the wall is noteworthy in cardiovascular fluid dynamics research as it characterizes the force exercised by the fluid tangential to a surface in contact with it. Shear stress at the wall is a substantial factor of endothelial role and plays a vibrant part in atherogenesis. Increased wall shear stress leads to an aneurysm and rupture. Overall, the shear stress at the wall signposts the flow situations in blood circulation; specifically, it relates to the impact on the vascular cell mechanisms. The axial variation of wall shear stress with respect to plasma layer thickness $\left(h_{P}\right)$ and maximum stenotic height $\left(\delta_{s}\right)$ for a Newtonian fluid $\left(\lambda_{1}=0\right)$ and Jeffrey fluid $\left(\lambda_{1}=1\right)$ has been presented, respectively, in figures 7 and

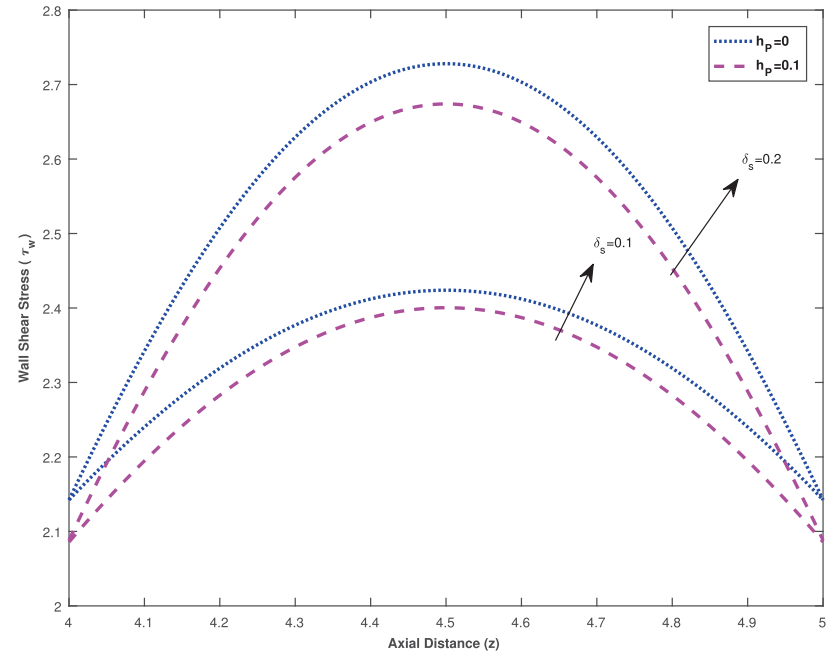

Figure 8. Axial variation of wall shear stress $\left(\tau_{w}\right)$ with plasma layer thickness $\left(h_{P}\right)$ for a non-Newtonian (Jeffrey) fluid $\left(\lambda_{1}=1\right)$.

8. It shows that the wall shear stress increases as the maximum stenotic height increases, but it decreases by the introduction of plasma layer near the Brinkman region. The presence of plasma layer between the core and Brinkman regions and the rheology of blood that obeys the law of a Jeffrey fluid have a remarkable effect on reducing the wall shear stress in the case of diseased blood vessels. When one moves from uniform region to non-uniform region, shear stress at the wall tends to increase; it attains a maximum at the mid-point of the stenosis, and begins to decrease in the downstream of the flow region irrespective of whether blood behaves as a Newtonian or non-Newtonian fluid. Another notable observation is that the introduction of plasma layer plays a predominant role in plummeting the shear stress at the wall in the case of blood as Newtonian fluid (figure 7).

Figure 9 is drawn to analyse the effect of concentration of blood cells (haematocrit) on the axial variation of wall shear stress. Haematocrit is the vital factor that governs the blood viscosity in the core region. We observe that an increase in haematocrit $\left(h_{m}\right)$ leads to increase in the wall shear stress as expected and its percentage of increase is found to be more significant for a lower value of haematocrit $\left(h_{m}\right)$. Figure 10 exhibits the combined influences of the thickness of Darcy region $\left(h_{D}\right)$ and Brinkman region $\left(h_{B}\right)$ on the wall shear stress. As the value of $h_{D}$ increases from 0.2 to 0.6 , the wall shear stress is increased, and its percentage increase or decrease along the axial direction (z) is higher for $h_{D}=0.6$. It is of interest to mention that when the value of $h_{D}>0.6$ the increase in the thickness of Darcy region $\left(h_{D}=0.6\right)$ significantly reduces the wall shear stress. Hence, the combined pertinent role of biophysical parameters $\left(h_{D}\right.$ and $\left.h_{B}\right)$ could be given appropriate prominence in updating the blood flow model.

Figure 11 displays the axial variation of wall shear stress with Darcy number $\left(D_{a}\right)$ and Darcy slip parameter $(\alpha)$. It is 


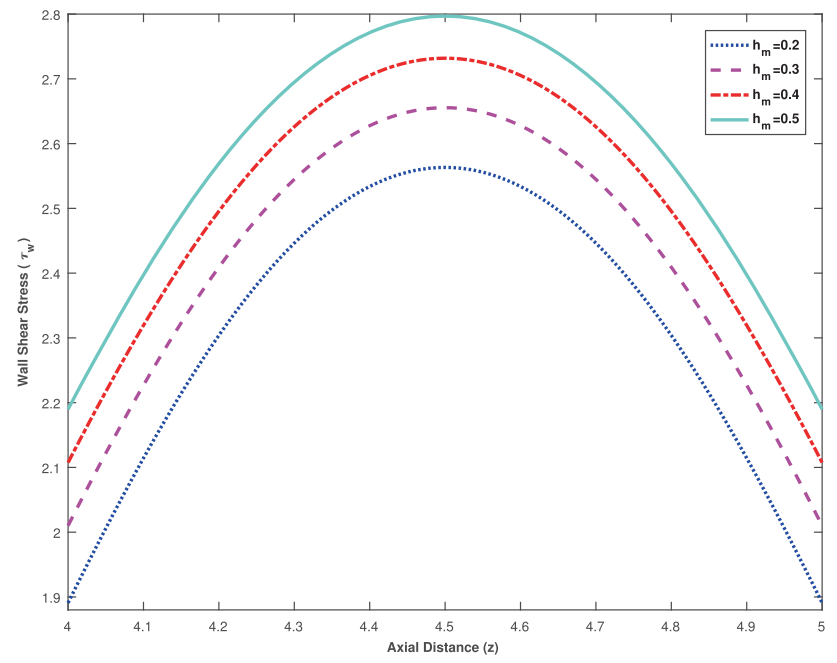

Figure 9. Axial distribution of wall shear stress $\left(\tau_{w}\right)$ with haematocrit $\left(h_{m}\right)$ in the stenotic region.

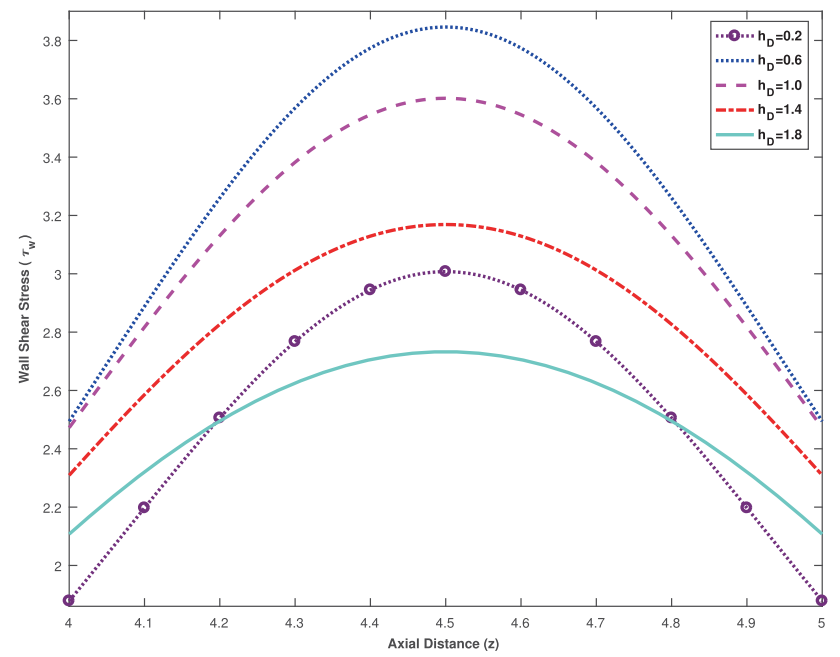

Figure 10. Wall shear stress $\left(\tau_{w}\right)$ distribution in the stenotic region for different values of the thickness of Darcy region $\left(h_{D}\right)$.

recorded from the results that the wall shear stress decreases with the increase in the values of $D_{a}$ and $\alpha$. It shows that the permeability of the arterial stenosis plays a predominant role in reducing the magnitude of wall shear stress as compared with the Darcy slip parameter. The significance of Jeffrey fluid parameter along with the plasma layer thickness on the axial variation of shear stress at the wall can be observed from figure 12. For a given value of Jeffrey fluid parameter $\left(\lambda_{1}\right)$ and plasma layer thickness $\left(h_{P}\right)$, the wall shear stress tends to increase or decrease in relation to increase or decrease in cross-sectional area in the diseased arterial stenosis. In case of the flow of Newtonian fluid in a porous arterial stenosis $\left(\lambda_{1}=0\right)$, the rate of decrease of the wall shear stress at $z=4.5$ (maximum depth of stenosis) is $7.51 \%$ approximately in the presence of peripheral plasma layer whereas it

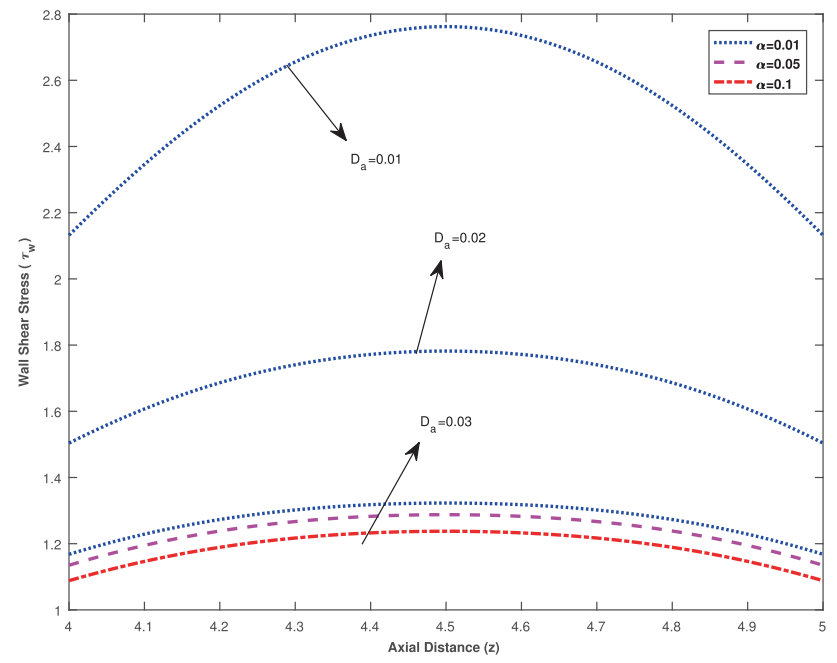

Figure 11. Distribution of wall shear stress $\left(\tau_{w}\right)$ in the stenotic region for different values of Darcy number $\left(D_{a}\right)$ and Darcy slip parameter $(\alpha)$.

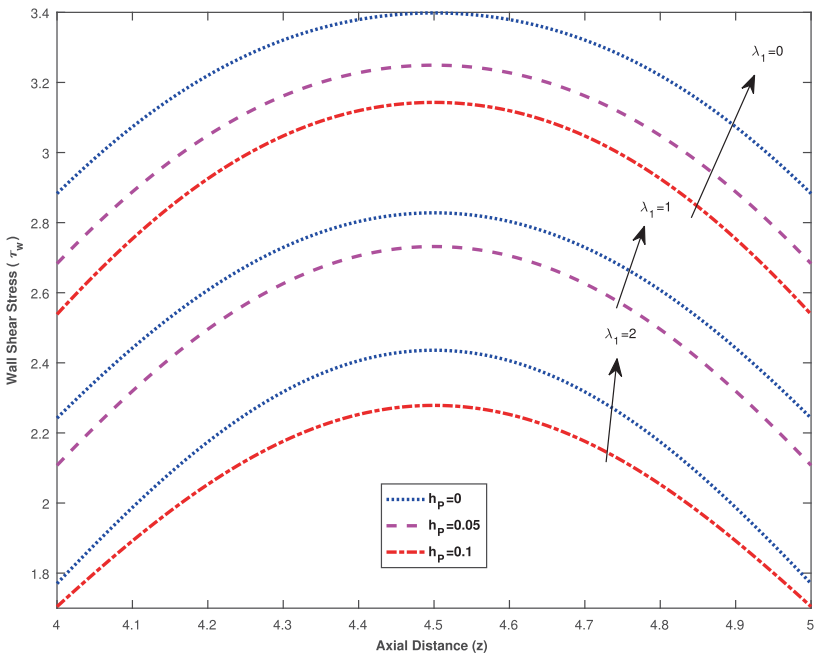

Figure 12. Axial variation of wall shear stress $\left(\tau_{w}\right)$ for different values of Jeffrey fluid parameter $\left(\lambda_{1}\right)$ and plasma layer thickness $\left(h_{P}\right)$ in the stenotic region.

turns out to be $6.46 \%$ for the same flow phenomenon of Jeffrey fluid having $\lambda_{1}=2.0$.

Figure 13 highlights the effects of Darcy number and Darcy slip parameter on the axial variation of flow resistance when the values of all other parameters are held fixed. It is easy to note from figure 13 that for increasing values of Darcy number $\left(D_{a}\right)$, the flow resistance decreases. A similar trend has been observed for the increase of Darcy slip parameter $(\alpha)$. Further, Darcy slip parameter seems to be a weak parameter as compared with the Darcy number in the sense that the former parameter has a tendency to bring a smaller decrease in the magnitude of the resistive impedance for the values of the parameters considered in the present investigation. Figure 14 illustrates that the flow 


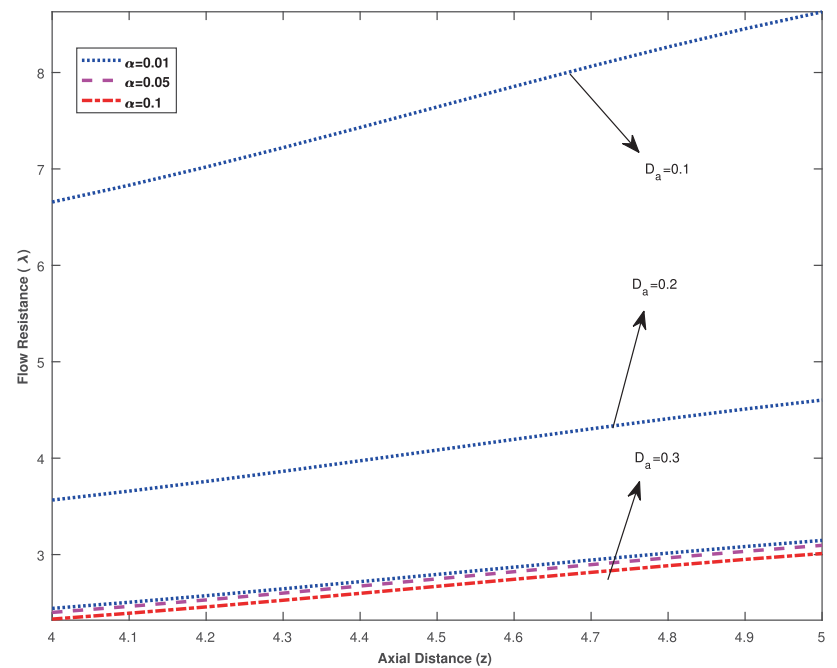

Figure 13. Axial distribution of flow impedance $(\lambda)$ with Darcy number $\left(D_{a}\right)$ and Darcy slip parameter $(\alpha)$ in the stenotic region.

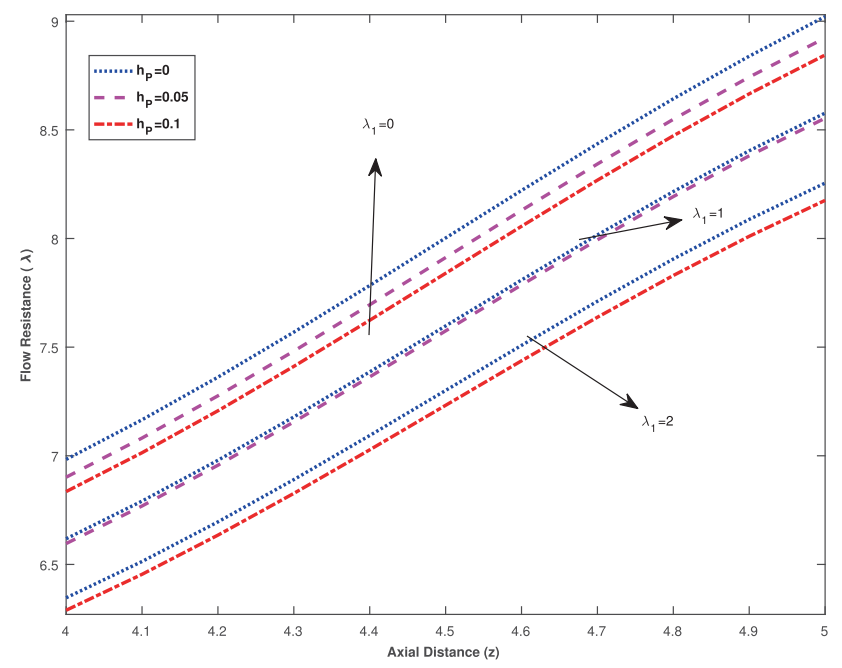

Figure 14. Axial distribution of flow impedance $(\lambda)$ with Jeffrey fluid parameter $\left(\lambda_{1}\right)$ and plasma layer thickness $\left(h_{P}\right)$ in the stenotic region.

resistance $(\lambda)$ increases with the increase in the axial distance $(z)$ and it is decreased by the introduction of plasma layer and the non-Newtonian behaviour of blood as Jeffrey fluid. For Newtonian fluid flow in porous arterial stenosis $\left(\lambda_{1}=0\right)$, the percentage decrease in the flow impedance at the end of the stenotic region is $1.959 \%$ approximately due to the presence of peripheral plasma layer whereas it becomes $0.963 \%$ for the same flow phenomena of Jeffrey fluid having $\lambda_{1}=2.0$.

The results of axial variation of resistive force (flow resistance or resistive impedance) for different values of haematocrit (concentration of blood cells) and the parameter $\eta$ are examined and displayed in figure 15. It is seen that the resistive force increases as the value of haematocrit $\left(h_{m}\right)$ increases, which is further provoked by increasing the

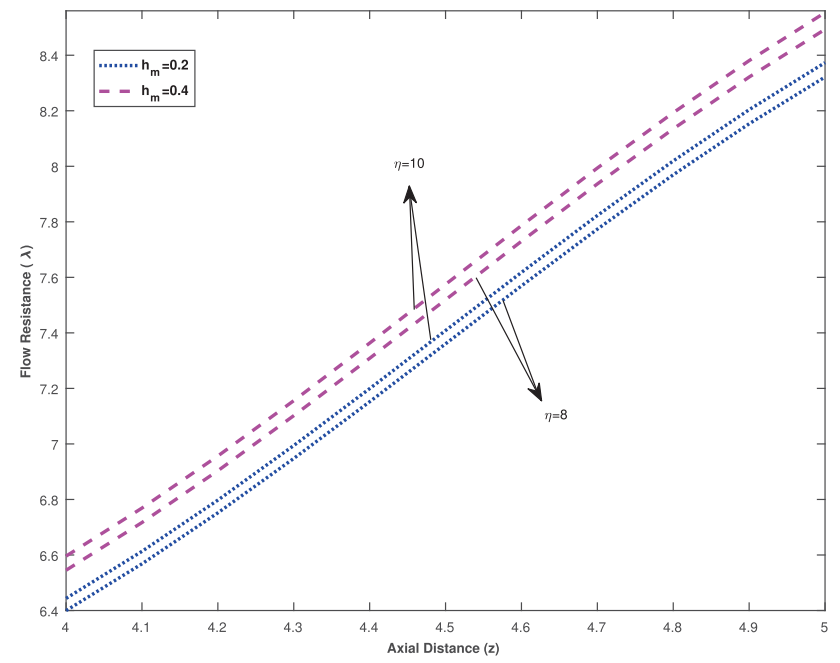

Figure 15. Flow resistance $(\lambda)$ distribution in the stenotic region for different values of $\eta$ and haematocrit $\left(h_{m}\right)$.

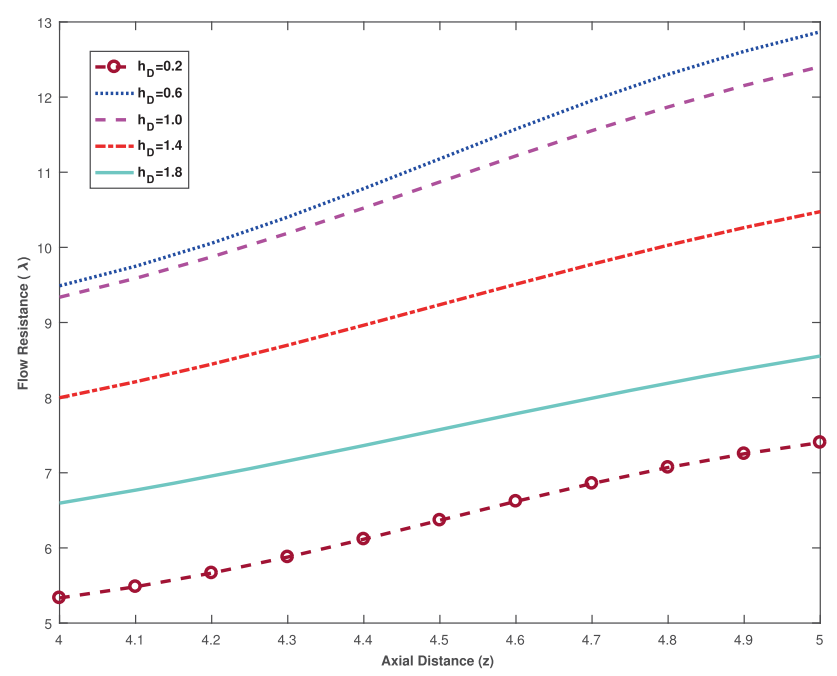

Figure 16. Variation of resistance to flow ( $\lambda$ ) with thickness of Darcy region $\left(h_{D}\right)$ in the stenotic region.

parameter $\eta$ involved in the expression of axially and radially variable core viscosity. This behaviour is attributed to the fact that the increase in $h_{m}$ and $\eta$ leads to increase of the viscous force in the core region, which makes the blood flow to experience a higher resistive force. Figure 16 is sketched to explore the combined impact of the thickness of Darcy region $\left(h_{D}\right)$ and Brinkman region $\left(h_{B}\right)$ on the flow resistance. For a lower value of $h_{D}$, the rate of increase in the resistive impedance with the increase in $z$ is found to be higher when one moves from the mid-point of the stenotic region to end of the stenotic region. As the value of $h_{D}$ increases from 0.2 to 0.6 , the flow resistance is increased. For $h_{D}>0.6$, it is remarkably noted that the flow resistance decreases as the thickness of Darcy region $\left(h_{D}\right)$ increases. Hence, the understanding of the combined relatable role of 


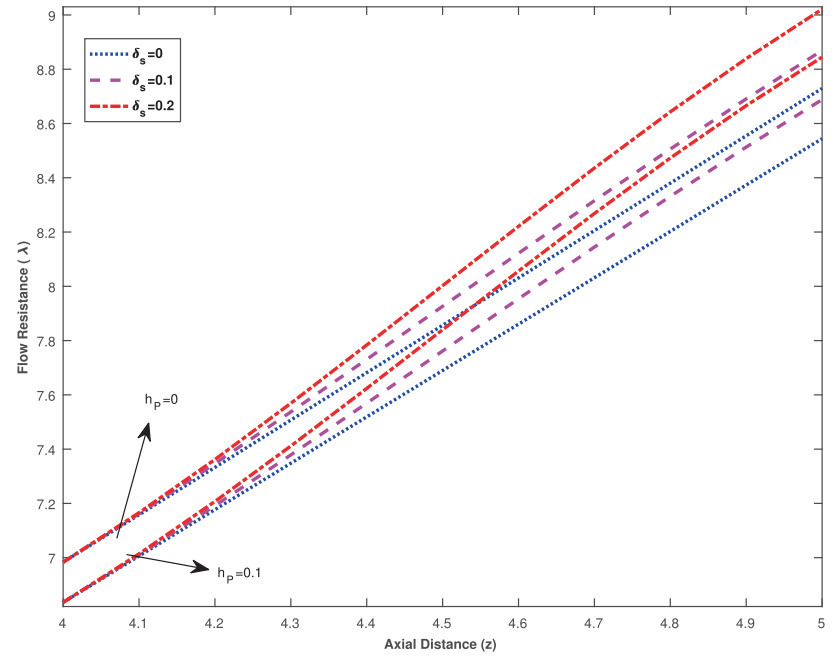

Figure 17. Variation of flow resistance $(\lambda)$ with stenotic height $\left(\delta_{s}\right)$ and plasma layer thickness $\left(h_{P}\right)$ for Newtonian fluid $\left(\lambda_{1}=0\right)$.

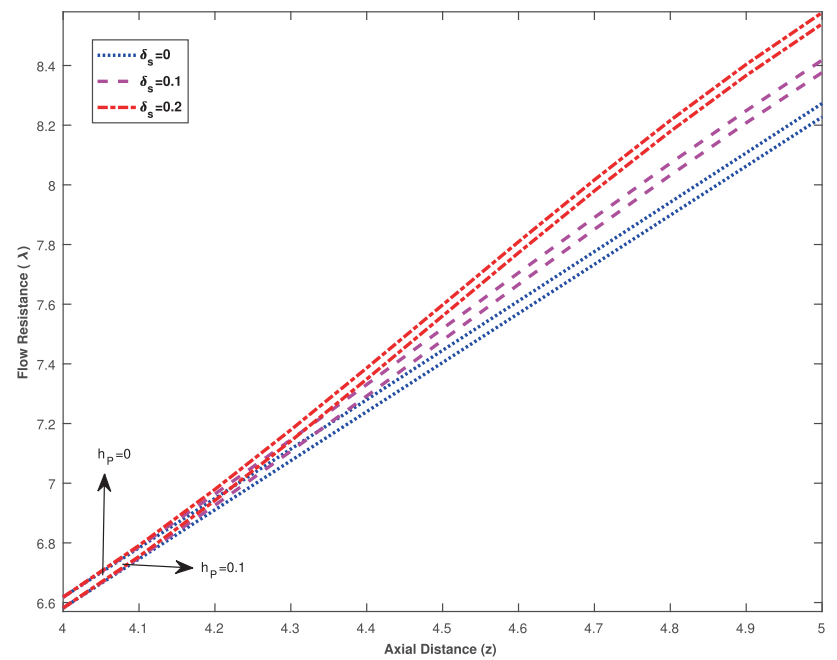

Figure 18. Variation of flow resistance $(\lambda)$ with stenotic height $\left(\delta_{s}\right)$ and plasma layer thickness $\left(h_{P}\right)$ for non-Newtonian (Jeffrey) fluid $\left(\lambda_{1}=1\right)$.

parameters $\left(h_{D}\right.$ and $\left.h_{B}\right)$ could be useful to enhance the required amount of blood supply to the vital organs (heart, liver, kidney and brain).

In figures 17 and 18, the combined role of Jeffrey fluid parameter $\left(\lambda_{1}\right)$, the maximum stenotic height $\left(\delta_{s}\right)$ and the plasma layer thickness $\left(h_{P}\right)$ in changing the pattern of the axial variation of resistive impedance $(\lambda)$ has been shown. For a fixed value of $\delta_{s}$ and $h_{P}$, flow impedance is less for a Jeffrey fluid, and it is higher for a Newtonian fluid. The introduction of plasma layer between the core and Brinkman regions significantly reduces the flow resistance whereas the presence of stenosis in an artery causes the blood flow to experience more resistive impedance. Figure 19 reveals that the flow resistance reduces with increase in stenosis shape parameter $(n)$ value and the amount of

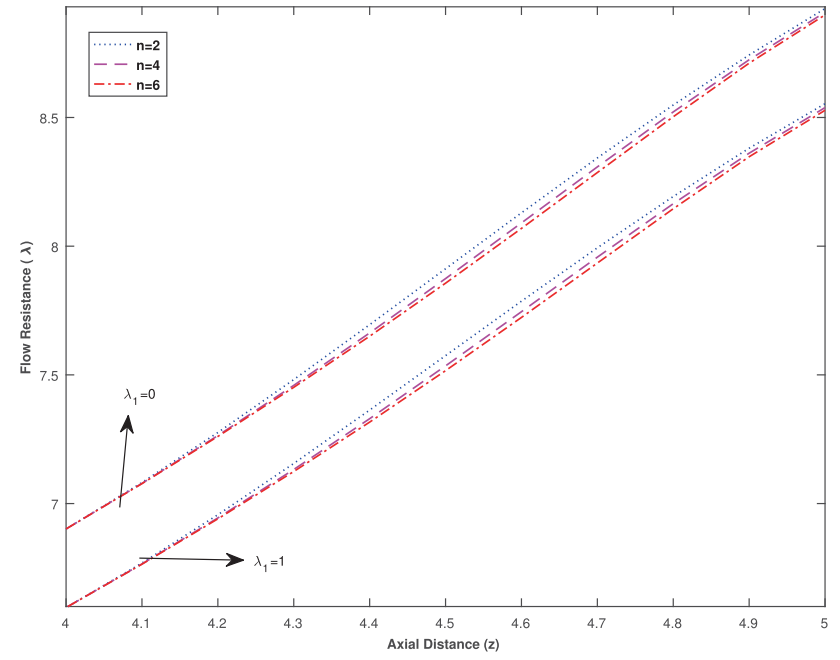

Figure 19. Axial variation of resistance to flow $(\lambda)$ for different values of stenosis shape parameter $(n)$.

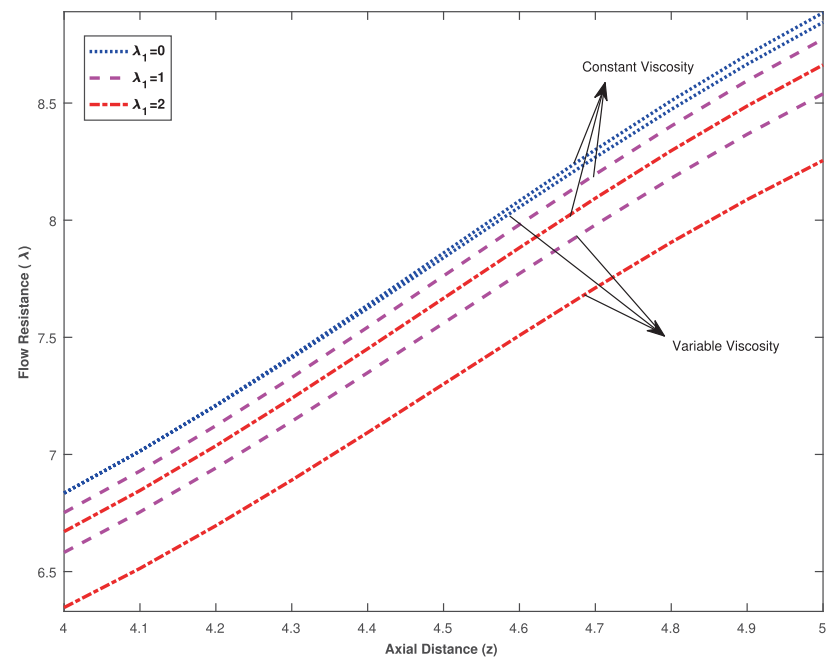

Figure 20. Variation of flow impedance $(\lambda)$ in the stenotic region with respect to constant and variable core viscosity.

flow resistance experienced by bloodstream is higher in the flow through an axially symmetrical stenosed tube than an axially non-symmetrical stenosed artery. The graphs in figure 20 have been plotted to highlight the influences of constant core fluid viscosity, and axially and radially variable core fluid viscosity on the flow resistance. Whether blood behaves like a Newtonian fluid or non-Newtonian fluid (Jeffrey fluid), the nature of axially and radially variable core fluid viscosity considered in the present investigation plays a predominant role in reducing the resistive impedance. The percentage of decrease in the resistive impedance due to the nature of axially and radially variable core fluid viscosity becomes approximately $1 \%, 3 \%$ and $5 \%$, respectively, for a Newtonian fluid $\left(\lambda_{1}=\right.$ $0)$ and Jeffrey fluid $\left(\lambda_{1}=1\right.$ and $\left.\lambda_{1}=2\right)$. This trend 
becomes higher for higher values of Jeffrey fluid parameter $\left(\lambda_{1}\right)$. It is pertinent to point out here that the amount of flow impedance experienced by the bloodstream is substantially less for the case of axially and radially variable core fluid viscosity.

\section{Conclusion}

The goal of the present mathematical analysis was to explore the collective effects of haematocrit (blood cells concentration), the plasma layer thickness between core and Brinkman regions, thickness of Darcy and Brinkman regions, non-Newtonian rheology of blood (Jeffrey fluid), and stenosis size and shape on the flow variables such as velocity profile, wall shear stress and flow resistance. To the best of the authors' knowledge, they have, for the first time, taken an effort to study the influences of the parameters on the flow characteristics. The physiologically important outcomes of the present work are summarized as follows:

1. It is, for the first time, observed that the axial velocity at the mid-point of the stenotic region decreases as the value of the thickness of Darcy region $\left(h_{D}\right)$ increases.

2. The magnitude of wall shear stress is significantly reduced by the introduction of plasma layer between the core and Brinkman regions and the rheology of blood obeying the law of a Jeffrey fluid.

3. The remarkable result is that for a lower value of the thickness of Darcy layer $\left(h_{D} \leq 0.6\right)$, the wall shear stress and flow impedance are increased with the increase in $h_{D}$, but they are found to be decreased for a higher value of $h_{D} \quad\left(0.6<h_{D} \leq 1.8\right)$, which forms the new findings added to the literature. We can conclude from this fact that a proper selection of the value of a thickness of Darcy layer could be useful to normalize the blood flow in diseased arterial stenosis and lead to the development of new diagnostic tools.

4. The wall shear stress and resistive impedance experienced by the flowing blood are increased due to the increase in the concentration of blood cells or volume fraction density of cells in the core region $\left(h_{m}\right)$ and the parameter $\eta$ involved in the expression of variable core viscosity.

5. The magnitudes of shear stress at the wall and flow resistance are significantly reduced by the non-Newtonian behaviour of blood as a Jeffrey fluid.

6. One of the most important outcomes of the present analysis is that the nature of axially and radially variable core fluid viscosity considered in the present investigation plays a pivotal role in reducing the resistive impedance.

Although the present work enlightens the effects of nonNewtonian rheology on the flow variables, non-symmetric stenosis, thickness of plasma layer and the porous wall, a modest effort would be made in future to study the flow of blood by considering the effects of deformable arteries, pulsatile nature of blood flow and walls, permeability of blood vessels, yield stress of the blood, viscoelastic nature of red blood cells and arterial wall, tapering of the blood vessels, curvature, branching, dilatation, and magnetic and electric fields, which could represent the real flow phenomenon.

\section{Acknowledgements}

Ramakrishna Manchi is thankful to the MHRD, the Government of India, for the grant of Fellowship for the Research Scholars administered by NIT, Tiruchirappalli.

\section{Nomenclatures}

$\bar{r}$

$\bar{z}$

$\bar{R}(\bar{z})$

$\bar{R}_{0}$

$\bar{d}$

$\bar{L}_{0}$

$\bar{L}$

$n$

$p_{i}(i=C, P, B, D)$

$P_{0}$

$R_{C}(z)$

$R_{P}(z)$

$R_{B}(z)$

$R_{D}(z)$

$h_{P}$

$h_{B}$

$h_{D}$

$u_{C}$

$u_{P}$

$u_{B}$

$u_{D}$

$h_{m}$

$D_{a}$

$I_{0}, K_{0}$

$Q$ represents dimensional quantities radial distance

axial distance

radius of the artery in stenosed region radius of the normal artery

location of stenosis

length of stenosis

length of the artery

shape parameter of stenosis

pressure

pressure gradient

radius of core region

radius of plasma region

radius of Brinkman region

radius of Darcy region

thickness of plasma layer

thickness of Brinkman layer

thickness of Darcy layer

velocity of fluid in core region

velocity of fluid in Plasma region

velocity of fluid in Brinkman region

velocity of fluid in Darcy region

haematocrit

Darcy number

modified Bessel functions

total flow rate

\section{Greek symbols}

$\delta_{s} \quad$ maximum height of stenosis

$\bar{\mu}_{C} \quad$ viscosity of Jeffrey fluid

$\bar{\mu}_{N}$ viscosity of Newtonian fluid

$\alpha_{2}$ effective viscosity of Brinkman layer

$\lambda_{1}$ ratio of relaxation to retardation times

$\alpha \quad$ Darcy slip parameter

$\beta \quad$ stress jump parameter

$\phi \quad$ porosity

$\tau_{w} \quad$ wall shear stress 
$\lambda$ flow impedance

\section{Appendix I}

$$
\begin{aligned}
& C_{2}=\left(4 \alpha \sigma I_{1}\left(\sigma R_{P}\right) K_{0}\left(\sigma R_{B}\right) P_{0} D_{a}^{3 / 2}\right. \\
& +4 \alpha \sigma I_{0}\left(\sigma R_{B}\right) K_{1}\left(\sigma R_{P}\right) P_{0} D_{a}^{3 / 2} \\
& +4 \sigma^{2} I_{1}\left(\sigma R_{P}\right) K_{1}\left(\sigma R_{B}\right) P_{0} D_{a}^{2} \\
& -4 \sigma^{2} I_{1}\left(\sigma R_{B}\right) K_{1}\left(\sigma R_{P}\right) P_{0} D_{a}^{2} \\
& -\sigma^{2} I_{1}\left(\sigma R_{P}\right) K_{1}\left(\sigma R_{B}\right) P_{0} R_{C}^{2} D_{a} \\
& +\sigma^{2} I_{1}\left(\sigma R_{B}\right) K_{1}\left(\sigma R_{P}\right) P_{0} R_{C}^{2} D_{a} \\
& +\sigma^{2} I_{1}\left(\sigma R_{P}\right) K_{1}\left(\sigma R_{B}\right) P_{0} R_{P}^{2} D_{a} \\
& -\sigma^{2} I_{1}\left(\sigma R_{B}\right) K_{1}\left(\sigma R_{P}\right) P_{0} R_{P}^{2} D_{a} \\
& -2 \sigma \phi I_{1}\left(\sigma R_{B}\right) K_{0}\left(\sigma R_{P}\right) P_{0} R_{P} D_{a} \\
& -2 \sigma \phi I_{0}\left(\sigma R_{P}\right) K_{1}\left(\sigma R_{B}\right) P_{0} R_{P} D_{a} \\
& -\alpha \sigma I_{1}\left(\sigma R_{P}\right) K_{0}\left(\sigma R_{B}\right) P_{0} R_{C}^{2} \sqrt{D_{a}} \\
& +\beta \sigma \phi I_{1}\left(\sigma R_{B}\right) K_{0}\left(\sigma R_{P}\right) P_{0} R_{C}^{2} \sqrt{D_{a}} \\
& +\beta \sigma \phi I_{0}\left(\sigma R_{P}\right) K_{1}\left(\sigma R_{B}\right) P_{0} R_{C}^{2} \sqrt{D_{a}} \\
& -\alpha \sigma I_{0}\left(\sigma R_{B}\right) K_{1}\left(\sigma R_{P}\right) P_{0} R_{C}^{2} \sqrt{D_{a}} \\
& +\alpha \sigma I_{1}\left(\sigma R_{P}\right) K_{0}\left(\sigma R_{B}\right) P_{0} R_{P}^{2} \sqrt{D_{a}} \\
& \text { - } \beta \sigma \phi I_{1}\left(\sigma R_{B}\right) K_{0}\left(\sigma R_{P}\right) P_{0} R_{P}^{2} \sqrt{D_{a}} \\
& -\beta \sigma \phi I_{0}\left(\sigma R_{P}\right) K_{1}\left(\sigma R_{B}\right) P_{0} R_{P}^{2} \sqrt{D_{a}} \\
& +\alpha \sigma I_{0}\left(\sigma R_{B}\right) K_{1}\left(\sigma R_{P}\right) P_{0} R_{P}^{2} \sqrt{D_{a}} \\
& -2 \alpha \phi I_{0}\left(\sigma R_{P}\right) K_{0}\left(\sigma R_{B}\right) P_{0} R_{P} \sqrt{D_{a}} \\
& +2 \alpha \phi I_{0}\left(\sigma R_{B}\right) K_{0}\left(\sigma R_{P}\right) P_{0} R_{P} \sqrt{D_{a}} \\
& +\alpha \beta \phi I_{0}\left(\sigma R_{P}\right) K_{0}\left(\sigma R_{B}\right) P_{0} R_{C}^{2} \\
& -\alpha \beta \phi I_{0}\left(\sigma R_{B}\right) K_{0}\left(\sigma R_{P}\right) P_{0} R_{C}^{2} \\
& -\alpha \beta \phi I_{0}\left(\sigma R_{P}\right) K_{0}\left(\sigma R_{B}\right) P_{0} R_{P}^{2} \\
& \left.+\alpha \beta \phi I_{0}\left(\sigma R_{B}\right) K_{0}\left(\sigma R_{P}\right) P_{0} R_{P}^{2}\right) / \\
& \left(4 \left(I_{1}\left(\sigma R_{P}\right) K_{1}\left(\sigma R_{B}\right) D_{a} \sigma^{2}\right.\right. \\
& -I_{1}\left(\sigma R_{B}\right) K_{1}\left(\sigma R_{P}\right) D_{a} \sigma^{2} \\
& +\alpha I_{1}\left(\sigma R_{P}\right) K_{0}\left(\sigma R_{B}\right) \sqrt{D_{a}} \sigma \\
& -\beta \phi I_{1}\left(\sigma R_{B}\right) K_{0}\left(\sigma R_{P}\right) \sqrt{D_{a}} \sigma \\
& -\beta \phi I_{0}\left(\sigma R_{P}\right) K_{1}\left(\sigma R_{B}\right) \sqrt{D_{a}} \sigma \\
& +\alpha I_{0}\left(\sigma R_{B}\right) K_{1}\left(\sigma R_{P}\right) \sqrt{D_{a}} \sigma \\
& -\alpha \beta \phi I_{0}\left(\sigma R_{P}\right) K_{0}\left(\sigma R_{B}\right) \\
& \left.\left.+\alpha \beta \phi I_{0}\left(\sigma R_{B}\right) K_{0}\left(\sigma R_{P}\right)\right)\right) \text {. } \\
& C_{4}=\left(4 \alpha \sigma I_{1}\left(\sigma R_{P}\right) K_{0}\left(\sigma R_{B}\right) P_{0} D_{a}^{3 / 2}\right. \\
& +4 \alpha \sigma I_{0}\left(\sigma R_{B}\right) K_{1}\left(\sigma R_{P}\right) P_{0} D_{a}^{3 / 2} \\
& +4 \sigma^{2} I_{1}\left(\sigma R_{P}\right) K_{1}\left(\sigma R_{B}\right) P_{0} D_{a}^{2}
\end{aligned}
$$

$$
\begin{aligned}
& -4 \sigma^{2} I_{1}\left(\sigma R_{B}\right) K_{1}\left(\sigma R_{P}\right) P_{0} D_{a}^{2} \\
& +\sigma^{2} I_{1}\left(\sigma R_{P}\right) K_{1}\left(\sigma R_{B}\right) P_{0} R_{P}^{2} D_{a} \\
& -\sigma^{2} I_{1}\left(\sigma R_{B}\right) K_{1}\left(\sigma R_{P}\right) P_{0} R_{P}^{2} D_{a} \\
& -2 \sigma \phi I_{1}\left(\sigma R_{B}\right) K_{0}\left(\sigma R_{P}\right) P_{0} R_{P} D_{a} \\
& -2 \sigma \phi I_{0}\left(\sigma R_{P}\right) K_{1}\left(\sigma R_{B}\right) P_{0} R_{P} D_{a} \\
& +\alpha \sigma I_{1}\left(\sigma R_{P}\right) K_{0}\left(\sigma R_{B}\right) P_{0} R_{P}^{2} \sqrt{D_{a}} \\
& -\beta \sigma \phi I_{1}\left(\sigma R_{B}\right) K_{0}\left(\sigma R_{P}\right) P_{0} R_{P}^{2} \sqrt{D_{a}} \\
& -\beta \sigma \phi I_{0}\left(\sigma R_{P}\right) K_{1}\left(\sigma R_{B}\right) P_{0} R_{P}^{2} \sqrt{D_{a}} \\
& +\alpha \sigma I_{0}\left(\sigma R_{B}\right) K_{1}\left(\sigma R_{P}\right) P_{0} R_{P}^{2} \sqrt{D_{a}} \\
& -2 \alpha \phi I_{0}\left(\sigma R_{P}\right) K_{0}\left(\sigma R_{B}\right) P_{0} R_{P} \sqrt{D_{a}} \\
& +2 \alpha \phi I_{0}\left(\sigma R_{B}\right) K_{0}\left(\sigma R_{P}\right) P_{0} R_{P} \sqrt{D_{a}} \\
& -\alpha \beta \phi I_{0}\left(\sigma R_{P}\right) K_{0}\left(\sigma R_{B}\right) P_{0} R_{P}^{2} \\
& \left.+\alpha \beta \phi I_{0}\left(\sigma R_{B}\right) K_{0}\left(\sigma R_{P}\right) P_{0} R_{P}^{2}\right) / \\
& \left(4 \left(I_{1}\left(\sigma R_{P}\right) K_{1}\left(\sigma R_{B}\right) D_{a} \sigma^{2}\right.\right. \\
& -I_{1}\left(\sigma R_{B}\right) K_{1}\left(\sigma R_{P}\right) D_{a} \sigma^{2} \\
& +\alpha \sigma I_{1}\left(\sigma R_{P}\right) K_{0}\left(\sigma R_{B}\right) \sqrt{D_{a}} \\
& -\beta \sigma \phi I_{1}\left(\sigma R_{B}\right) K_{0}\left(\sigma R_{P}\right) \sqrt{D_{a}} \\
& -\beta \sigma \phi I_{0}\left(\sigma R_{P}\right) K_{1}\left(\sigma R_{B}\right) \sqrt{D_{a}} \\
& +\alpha \sigma I_{0}\left(\sigma R_{B}\right) K_{1}\left(\sigma R_{P}\right) \sqrt{D_{a}} \\
& -\alpha \beta \phi I_{0}\left(\sigma R_{P}\right) K_{0}\left(\sigma R_{B}\right) \\
& \left.\left.+\alpha \beta \phi I_{0}\left(\sigma R_{B}\right) K_{0}\left(\sigma R_{P}\right)\right)\right) \\
& C_{5}=\phi P_{0} \sqrt{D_{a}}\left(\alpha K_{0}\left(\sigma R_{B}\right)\right. \\
& \left.+\sigma K_{1}\left(\sigma R_{B}\right) \sqrt{D_{a}}\right)\left(R_{P}-2 \beta \sqrt{D_{a}}\right) / \\
& 2\left[\left(\beta \phi I_{0}\left(\sigma R_{P}\right)\right.\right. \\
& \left.-\sigma \sqrt{D_{a}} I_{1}\left(\sigma R_{P}\right)\right)\left(\alpha K_{0}\left(\sigma R_{B}\right)\right. \\
& \left.+\sigma \sqrt{D_{a}} K_{1}\left(\sigma R_{B}\right)\right) \\
& -\left(\alpha I_{0}\left(\sigma R_{B}\right)-\sigma \sqrt{D_{a}} I_{1}\left(\sigma R_{B}\right)\right)\left(\beta \phi K_{0}\left(\sigma R_{P}\right)\right. \\
& \left.\left.+\sigma \sqrt{D_{a}} K_{1}\left(\sigma R_{P}\right)\right)\right] \text {. } \\
& C_{6}=\phi P_{0} \sqrt{D_{a}}\left(\sigma \sqrt{D_{a}} I_{1}\left(\sigma R_{B}\right)\right. \\
& \left.+-\alpha I_{0}\left(\sigma R_{B}\right)\right)\left(2 \beta \sqrt{D_{a}}-R_{P}\right) / \\
& 2\left[-D_{a} \sigma^{2} I_{1}\left(\sigma R_{P}\right) K_{1}\left(\sigma R_{B}\right)\right. \\
& ++D_{a} \sigma^{2} I_{1}\left(\sigma R_{B}\right) K_{1}\left(\sigma R_{P}\right) \\
& -\alpha \sigma \sqrt{D_{a}} I_{1}\left(\sigma R_{P}\right) K_{0}\left(\sigma R_{B}\right) \\
& +\sigma \beta \phi \sqrt{D_{a}} I_{1}\left(\sigma R_{B}\right) K_{0}\left(\sigma R_{P}\right) \\
& +\sigma \beta \phi \sqrt{D_{a}} I_{0}\left(\sigma R_{P}\right) K_{1}\left(\sigma R_{B}\right) \\
& -\alpha \sigma \sqrt{D_{a}} I_{0}\left(\sigma R_{B}\right) K_{1}\left(\sigma R_{P}\right) \\
& +\alpha \beta \phi I_{0}\left(\sigma R_{P}\right) K_{0}\left(\sigma R_{B}\right) \\
& \left.+-\alpha \beta \phi I_{0}\left(\sigma R_{B}\right) K_{0}\left(\sigma R_{P}\right)\right] \text {. }
\end{aligned}
$$




\section{References}

[1] Young D F 1968 Effects of a time-dependent stenosis on flow through a tube. Trans. ASME J. Eng. Ind. 90: 248-254

[2] Boyd W 1961 Text book of pathology: structure and functions in diseases. Philadelphia: Lea and Fibiger

[3] Young D F and Tsai F Y 1973 Flow characteristics in models of arterial stenosis-I: steady flow. J. Biomech. 6: 395-410

[4] Macdonald D A 1979 On steady flow through modeled vascular stenosis. J. Biomech. 12: 13-20

[5] Chaturani P and Ponnalagarsamy R 1986 Pulsatile flow of Casson's fluid through stenosed arteries with applications to blood flow. Biorheology 23: 499-511

[6] Young D F 1979 Fluid mechanics of arterial stenosis. Trans. ASME J. Biomech. Eng. 101: 157-175

[7] Misra J C, Sinha A and Shit G C 2011 Mathematical modeling of blood flow in a porous vessel having double stenoses in the presence of an external magnetic field. Int. J. Biomath. 4: 207-225

[8] Charm S E and Kurland G 1965 Viscometry of human blood for shear rates of 0-100,000 $\mathrm{sec}^{-1}$. Nature 206: 617-618

[9] Whitmore R L 1968 Rheology of the circulation. New York: Pergamon Press

[10] Lih M 1975 Transport phenomena in medicine and biology. New York: Wiley

[11] Srivastava L M 1985 Flow of a couple-stress fluid through stenotic blood vessels. J. Biomech. 18: 479-485

[12] Akbar N S, Nadeem S and Ali M 2011 Jeffrey fluid model for blood flow through a tapered artery with a stenosis. $J$. Mech. Med. Biol. 11: 529-545

[13] Shah S R and Anamika 2017 Mathematical and computational study of blood flow through diseased artery. IPASJ Int. J. Comput. Sci. 5: 1-6

[14] Bugliarello G and Hayden J W 1963 Detailed characteristics of the flow of blood in vitro. Trans. Soc. Rheol. 7: 209-230

[15] Bugliarello G and Sevilla J 1970 Velocity distribution and other characteristics of steady and pulsatile blood flow in fine glass tubes. Biorheology 7: 85-107

[16] Cokelet G R 1972 The rheology of human blood. In: Biomechanics . Englewood Cliffs, N.J.: Prentice-Hall

[17] Shukla J B, Parihar R S and Rao B R P 1980 Effects of stenosis on non-Newtonian flow of the blood in an artery. Bull. Math. Biol. 42: 283-294

[18] Chaturani P and Ponnalagarsamy R 1982 A two layered model for blood flow through stenosed arteries. In: Proceedings of the 11th National Conference on Fluid Mechanics and Fluid Power, pp. 16-22

[19] Priyadharshini S and Ponalagusamy R 2017 Computational model on pulsatile flow of blood through a tapered arterial stenosis with radially variable viscosity and magnetic field. Sadhana 42: 1901-1913

[20] Nallapu S and Radhakrishnamacharya G 2014 Flow of Jeffrey fluid through narrow tubes. Int. J. Sci. Eng. Res. 4: 468-473

[21] Sharma B D, Yadav P K and Filippov A 2017 A Jeffrey-fluid model of blood flow in tubes with stenosis. Colloid J. 79: 849-856
[22] Ponalagusamy R 2016 Particulate suspension Jeffrey fluid flow in a stenosed artery with a particle-free plasma layer near the wall. Korea-Aust. Rheol. J. 28: 217-227

[23] Ponalagusamy R and Tamil Selvi R 2013 Blood flow in stenosed arteries with radially variable viscosity, peripheral plasma layer thickness and magnetic field. Meccanica 48: $2427-2438$

[24] Ponalagusamy R 2007 Blood flow through an artery with mild stenosis: a two-layered model, different shapes of stenoses and slip velocity at the wall. J. Appl. Sci. 7: 1071-1077

[25] Whitaker S Flow in porous media I: a theoretical derivation of Darcy's law. Transp. Porous Media 1: 3-25

[26] Brinkman H C Experimental data on the viscous force exerted by a flowing fluid on a dense swarm of particles. Appl. Sci. Res. A 1: 27-34

[27] Raj A and Sen A K 2016 Flow-induced deformation of compliant microchannels and its effect on pressure-flow characteristics. Microfluid. Nanofluid. 20: 1-13

[28] Raj A and Sen A K 2018 Entry and passage behavior of biological cells in a constricted compliant microchannel. RSC Adv. 8: 20884-20893

[29] Raj A, Halder R, Sajeesh P and Sen A K 2016 Droplet generation in a microchannel with a controllable deformable wall. Microfluid. Nanofluid. 20: 1-16

[30] Nallapu S, Radhakrishnamacharya G and Chamkha J 2015 Flow of a Jeffrey fluid through a porous medium in narrow tubes. J. Porous Media 18: 71-78

[31] Ponalagusamy R 2017 Two-fluid model for blood flow through a tapered arterial stenosis: effect of non-zero couple stress boundary condition at the interface. Int. J. Appl. Comput. Math. 3: 807-824

[32] Ponalagusamy R 1986 Blood flow through stenosed tube. PhD Thesis. Bombay, India: IIT

[33] Brown B A, Williams H and George S J 2017 Evidence for the involvement of Matrix-Degrading Metalloproteinases (MMPs) in atherosclerosis. Prog. Mol. Biol. Transl. Sci. 147: 197-237

[34] Goharzadeh A, Saidi A, Wang D, Merzkirch W and Khalili A 2006 An experimental investigation of the Brinkman layer thickness at a fluid-porous interface. In: Proceedings of the IUTAM Symposium on One Hundred Years of Boundary Layer Research, pp. 445-454

[35] Hill A A and Straughan B 2008 Poiseuille flow in a fluid overlying a porous medium. J. Fluid Mech. 603: 137-149

[36] Boodoo C, Bhatt B and Comissiong D 2013 Two-phase fluid flow in a porous tube: a model for blood flow in capillaries. Rheol. Acta 52: 579-588

[37] Sharma B D and Yadav P K 2017 A two-layer mathematical model of blood flow in porous constricted blood vessels. Transp. Porous Media 120: 239-254

[38] Straughan B 2008 Stability and wave motion in porous media. New York: Springer

[39] Ochoa-Tapia J A and Whitakeri S 1995 Momentum transfer at the boundary between a porous medium and a homogeneous fluid I. Theoretical development. Int. J. Heat Mass Transf. 38: 2635-2646

[40] Beavers G S and Joseph D D 1967 Boundary conditions at a naturally permeable wall. J. Fluid Mech. 30: 197-207 